\title{
South Pole Neutron Monitor Sensitivity to Geomagnetic Field Variations
}

\author{
John Clem and Paul Evenson * \\ Bartol Research Institute, Department of Physics and Astronomy, University of Delaware \\ 217 Sharp Lab, University of Delaware, Newark, DE 19716, USA \\ E-mail: jmc@udel.edu and evenson@udel.edu
}

Secular variation of the Earth's geomagnetic fields is well known to change the cutoff rigidity, and thereby the count rate of low latitude neutron monitors. Such changes are generally assumed to be irrelevant to so called atmosphere limited neutron monitors at high latitudes. We have documented a secular change in the count rate of the neutron monitor at Amundsen - Scott Station, located at the geographic South Pole. This monitor is conventionally assumed to be atmosphere limited, but the combination of low cutoff and high altitude is unique within the worldwide network of monitors. We are constructing a more accurate model of secondary particle production in this environment. Using this model we present preliminary results on the sensitivity of the South Pole Neutron Monitor to changes in the geomagnetic field over a 40 year period.

The 34th International Cosmic Ray Conference

30 July- 6 August, 2015

The Hague, The Netherlands

*Speaker. 


\section{Introduction}

The neutron monitor at the Amundsen Scott Station, located at the geographic South Pole, has operated with some interruptions since 1964. Particles detected by such monitors are secondary cosmic rays, byproducts of nuclear and high energy interactions of primary cosmic rays (predominantly protons and helium nuclei) with Earth's atmosphere. The neutron monitor counting rate at South Pole follows an 11 year cycle with maxima at times of low solar activity, but over the entire interval exhibits an apparently steady decline, totaling approximately $10 \%$ by 2013. We have worked extensively to identify possible instrumental or ground level environmental sources of this decline but to date have found none. In this paper we discuss the continuing decrease of the count rate with time and explore the question of whether the increasing cutoff at high zenith angle could influence the radiation environment at the surface of the polar ice cap.

An IGY type neutron monitor [Simpson, 2000] recorded data at the geographical South Pole from March 1964 until October 1974. Following a hiatus of more than 2 years, measurements resumed in January 1977 when a three-tube NM64 type monitor was installed at the station. This instrument operated until 22 November 2005, when operation was suspended. The detector tubes were removed and placed in heated storage, but much of the monitor superstructure (lead producer, polyethylene moderator, and reflector) remained in place at South Pole. In a retrospective analysis of the entire data set, Bieber et al. [2007] reported that the overall counting rate of the neutron monitor, corrected for equipment differences, declined by approximately $8 \%$ from 1965 to 1997. After considering many possible instrumental causes for the decline their ultimate conclusion was that it represented a true decline in the radiation environment at the detector. Discounting any effects due to the changing geomagnetic field, Bieber et al. [2007] proposed two possible, but still unlikely, causes for this decline. One was a significant change in the environment of the monitor as surrounding structures were slowly covered by snow while the monitor itself was repeatedly raised to stay above the snow surface. Another possibility was a subtle, secular change in the spectrum of cosmic rays in the rigidity range 1 to $3 \mathrm{GV}$. Because of its unique location and construction they showed that there was no other directly comparable detector available either to confirm or dispute such a possibility.

Reactivating the neutron monitor in February 2010, after a four-year, three month gap, allowed us to address directly our speculative causes for the long-term decline. Calibration before and after moving the main part of the monitor to a new location essentially excludes the environment as a cause. Response of the reactivated monitor to the unusually high cosmic ray fluxes at all energies in 2009 makes it less likely that a subtle change in the cosmic ray spectrum caused the decline. By all other measures the cosmic ray flux reached record levels in 2009 yet the monitor at Pole barely came back to its 1987 level. The secular decline appears real and indeed continuing. In this paper we discuss the beginning of a study to examine the sensitivity of the South Pole Neutron monitor to these changes in the geomagnetic cutoff as a possible cause of the decline.

To understand the motivation for our speculation that geomagnetic effects may be visible at the South Pole it is necessary to consider the origin of the counts in a neutron monitor. This is achieved utilizing the model described in Clem et al. [2000, 2004]. Such a calculation of the counting rate of a neutron monitor has four principal components: 
1) An assumed cosmic ray spectrum and composition outside the magnetosphere

2) A cutoff "sky map" that specifies the access of the cosmic rays through the magnetosphere

3) A model of the production and transport of secondary particles in the atmosphere

4) A model of the detector response to the secondary particles

Typically some well justified assumptions are made, based on the knowledge that the number of secondary particles reaching the surface falls off rapidly with the incident zenith angle of the primary. At sea level, even for vertical incidence, there are roughly seven nuclear interaction lengths between the top of the atmosphere and the surface. Thus the incident zenith angle dependence in the cutoff rigidity is typically truncated to 75 degrees. Primaries that satisfy this condition are run through the transport model in the atmosphere; primaries that do not satisfy the condition are ignored.
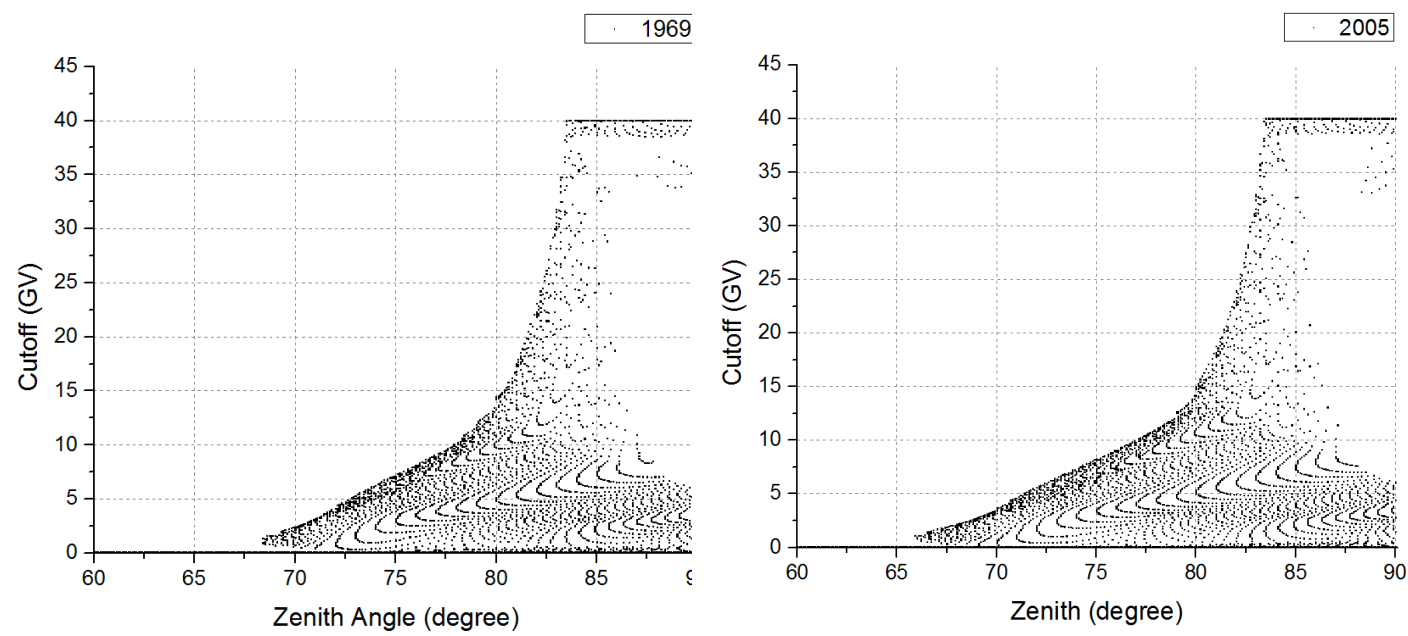

Figure 1: Geomagnetic cutoff at South Pole as a function of zenith angle for various azimuth angles calculated for 1969 and 2005.

Two properties of the unique location at the South Pole combine to cause us to think this approximation should be examined. First is the high altitude; for vertical incidence the surface is only about five interaction lengths from the top of the atmosphere. Particles incident at larger zenith angles can therefore contribute to the count rate. Second is the structure of the cutoff itself, illustrated in Figure 1. There is a small region of very high cutoff concentrated at large zenith angle. As the extent of this region is larger in 2005 than it is in 1969 there is likely to be some reduction in the counting rate, however it is not at all obvious is that the reduction will be significant.

\section{New Calculation}

What is obvious is that the truncated skymap is not appropriate for this calculation. This paper reports our initial progress in relaxing this assumption, where we have constructed a skymap extended to 90 degrees with higher angular resolution. We however maintain the definition of the skymap, applying it at every point in the spherical atmosphere in relation to the local zenith. The 
result of this calculation, using a typical primary spectrum, is shown in Figure 2. Indeed we obtain a decrease in the counting rate as a function of time, but the effect is tiny.

We can investigate the overall accuracy of this calculation by considering the time

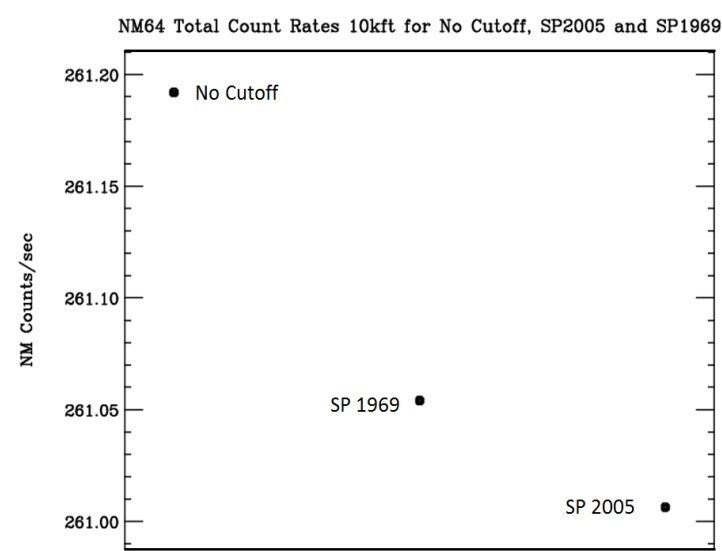

Figure 2: South Pole neutron monitor counting rate calculated for a spherical atmosphere and various assumptions for the geomagnetic cutoff.

dependence of the counting rate. In the left panel of Figure 3 we show a compilation of directly measured proton and helium spectra together with fits to the spectra using a simple propagation model. We do not discuss the model here because in this context we have only used it to produce continuous representations of the data [Clem, et al. 2004]. In the right panel we show our calculation of the count rate at these times using the fitted spectra compared with the measured counting rate. The overall agreement of the calculation and the measurement is at the $10 \%$ level.
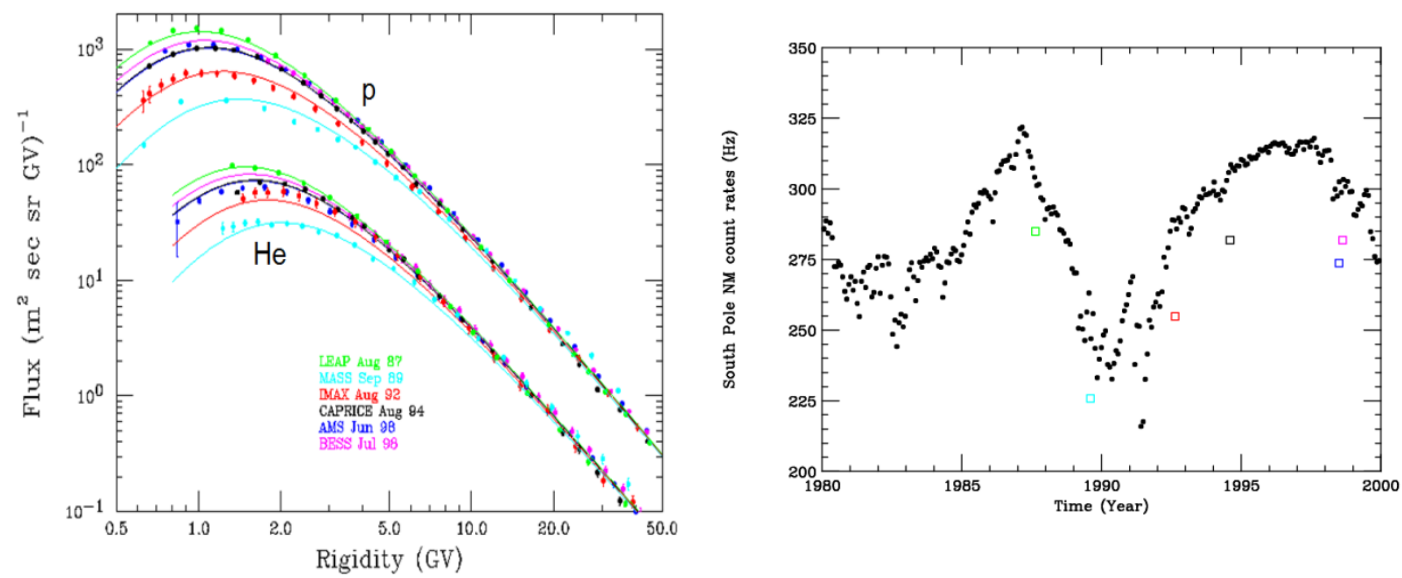

Figure 3 (Left) Measured proton and helium spectra at different times . (Right) Calculated South Pole neutron monitor counting rate at these times (open symbols) compared with the actual counting rate. 
Another way to compare the calculation and observation is shown in Figure 4, with the same data presented as a scatterplot. Note that the extrapolated best fit line shows a significant offset from the origin. The excess counting rate of the data compared to the calculation appears to be constant as a function of modulation level.

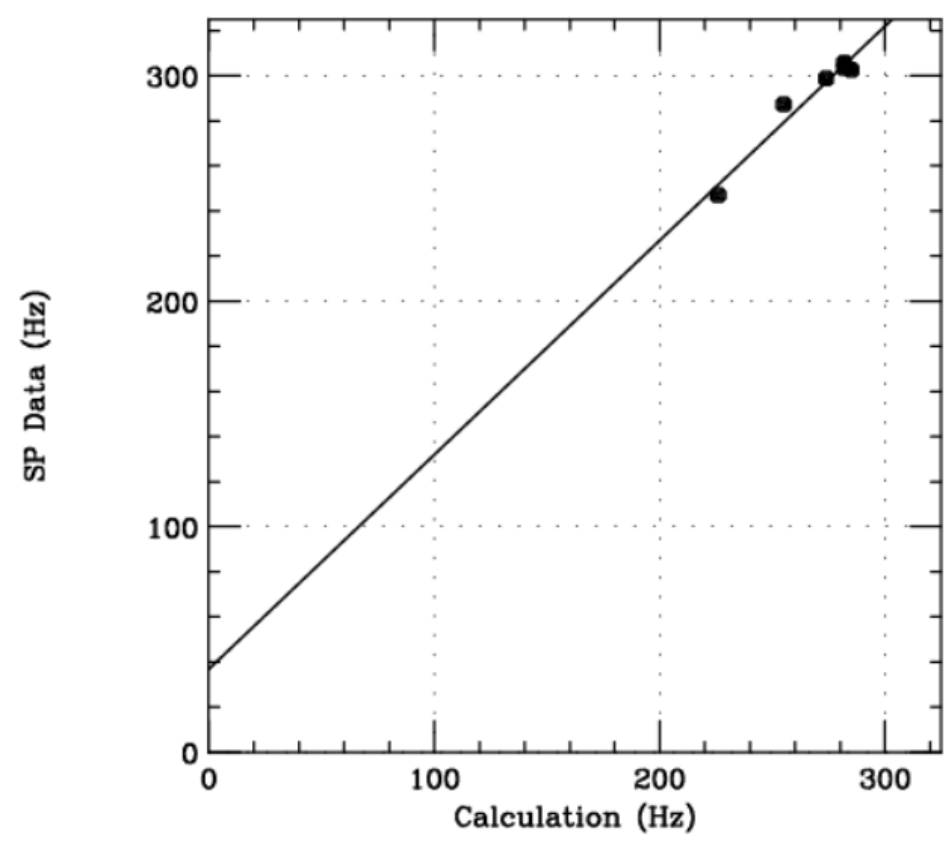

Figure 4: Actual South Pole compared to calculation shown as a scatterplot. Line is a fit to the data

\section{Discussion and Conclusions}

Incorporation of the extended skymaps in a calculation of the counting rate of the South Pole neutron monitor predicts a small decline in the counting rate as a function of time but it is far too small to explain the observed decline. Overall agreement of the calculation and the observed rate is good. Since the calculation under predicts the observation there is no immediate basis to conclude that some unseen high cutoff region is lurking somewhere waiting to be discovered.

It is potentially interesting that the error in the calculation is better expressed as an offset rather than a factor. If the discrepancy were due to a lack of knowledge of nuclear cross sections or secondary multiplicities one might expect the error to be better expressed as a factor. However if the error resides exclusively in the very high energy regime, the effect could manifest itself as a modulation independent offset. Excess observed counts could be a possible indication of an error in the assumed power index for the primary spectrum effecting exclusively the high energy component.

Although this simple model does not support the theory that secular variation of the Earth's geomagnetic field is the source of the observed decline, it does not necessarily disprove the idea. The case is not yet decided until we determine the source of the 10 percent effects not described by the present calculation. Our next project is to investigate the accuracy of the primary spectrum model using recent data from Pamela and AMS-2 instruments. Furthermore we plan to include the atmospheric magnetic field in the calculation and to treat the cutoff itself in more realistic terms. At the South Pole there is no Stoermer cutoff. The cutoff is entirely determined by stopping 
particles in the atmosphere. The primary that is excluded by this type of cutoff at location $\mathrm{X}$ has in fact entered the atmosphere at some other location Y.

\section{Acknowledgements}

We thank John Bieber and Roger Pyle for helpful discussions, and James Roth for his efforts as technical manager of the University of Delaware neutron monitor stations. Supported in part by United States National Science Foundation awards PLR-1341562 and PLR-1245939.

\section{References}

Bieber, J., et al., Long-term decline of South Pole neutron rates, 112, A12102, doi:10.1029/2006JA011894, 2007

Clem, J., et al., "Neutron Monitor Response Functions,” Space Science Reviews, volume 93, 335, 2000.

Clem, J., et al., "New calculations of the atmospheric cosmic radiation field—results for neutron spectra”, Radiat Prot Dosimetry, 110: 423-428, 2004.

Simpson, J. A., The cosmic ray nucleonic component: The invention and scientific uses of the neutron monitor, Space Sci. Rev., 93, 11 -32, 2000 